\title{
ESTRATEGIA PARA LA INFERENCIA CAUSAL Y PLANIFICACIÓN DE ESTUDIOS OBSERVACIONALES EN LAS CIENCIAS SOCIALES: EL CASO DE CHAITÉN POST ERUPCIÓN DEL 2008*
}

\author{
A Strategy for Causal Inference and the Planning of Observational \\ Studies in the Social Sciences: The Case of Chaitén after the Volcanic \\ Eruptions of 2008
}

\section{LUIS MALDONADO}

Instituto de Sociología, PUC (Chile)

\section{EDMUNDO KRONMÜLLER RIOSECO}

Escuela de Psicología, PUC (Chile)

\section{IGNACIO GUTIÉRREZ CROCCO}

Centro Nacional de Investigación para la

Gestión Integrada de Desastres (CIGIDEN)

\begin{abstract}
RESUMEN
El artículo presenta una estrategia para generar comparaciones válidas en estudios observacionales. Dicha estrategia es ilustrada a través del análisis del caso de Chaitén después de las erupciones volcánicas de 2008. Como producto de este desastre, la ciudad sufrió el desborde el río Blanco, por lo que quedó dividida entre los sectores norte y sur. El Estado de Chile declaró a Chaitén como inhabitable hasta el 2011, cuando se autorizó la ocupación solo del sector norte. Al día de hoy, ambos sectores han sido ocupados, lo que marca una fuerte diferencia entre asentamientos regulares e irregulares. Utilizamos dicha distinción para identificar el efecto de vivir en un asentamiento irregular en la confianza y cooperación. Para ello, y basándonos en la estrategia presentada, analizamos información cualitativa y datos de una encuesta aplicada a la población de Chaitén en 2015. El análisis de información cualitativa indica un significativo proceso de autoselección de los sujetos en las condiciones del tratamiento. Sobre la base de dichos análisis, diseñamos comparaciones válidas en base al método de Full Matching y analizamos los efectos sobre las variables dependientes de interés. Los resultados indican que vivir en asentamientos irregulares incrementaría, en promedio, la cooperación. Sin embargo, habría una fuerte heterogeneidad en la respuesta de los agentes a la intervención bajo análisis y sería necesario considerar factores no observados en los datos analizados para mejorar la comprensión de los resultados. Considerando la estrategia presentada y el caso de estudio discutido, desarrollamos una serie de implicancias para el diseño y planificación de estudios observacionales.
\end{abstract}

Luis Maldonado reconoce apoyo financiero del Centro Nacional de Investigación para la Gestión Integrada de Desastres Naturales (CIGIDEN) y del proyecto INICIO Número 17/2013, otorgado por la Vicerrectoría de Investigación (VRI) de la Pontificia Universidad Católica de Chile.

Edmundo Kronmüller reconoce apoyo financiero de Proyecto Fondecyt- Conicyt $\mathrm{N}^{\circ} 1141187$.

Agradecemos a los dos revisores anónimos por sus contribuciones a la mejora del texto. 
Palabras clave: plan de análisis, estudios observacionales, Chaitén, Full Matching

\begin{abstract}
This article presents a strategy to improve comparisons in observational studies. We illustrate this strategy by analyzing the case of Chaitén after the volcanic eruptions of 2008. As a result of this natural disaster, that city suffered the overflow of the Blanco River, leaving the city divided between the north and south sectors. The Chilean State declared Chaiten to be uninhabitable until 2011, where it exclusively authorized the occupation of the northern sector. To date, both sectors have been occupied, marking a sharp difference between regular and irregular settlements. We use this distinction to identify the effect that living in an irregular settlement has on trust and cooperation. In order to do this, we analyzed qualitative information and survey data from 2015. In a first step, the analysis of qualitative information indicates a significant process of self-selection of the subjects in the treatment conditions. Then, we designed valid comparisons based on a Full Matching method for analyzing the effects on the dependent variables. The results indicate that, on average, living in irregular settlements increased cooperation. However, there may be a strong heterogeneity in the way that the agents respond to the interventions here analyzed. As a consequence, in order to improve the interpretation of these results, it would be necessary to take into account other factors not observed in the data here analyzed. We finish this study by developing a series of implications for the design and planning of observational studies, in general.
\end{abstract}

Key words: analysis plan, observational studies, Chaitén, Full Matching 


\section{INTRODUCCIÓN}

Uno de los grandes objetivos de las Ciencias Sociales es establecer relaciones causales entre los fenómenos que estudian. Así, desde las teorías desarrolladas en la Sociología, la Psicología Social, la Economía y la Ciencia Política se desprenden hipótesis que vinculan causalmente dos fenómenos y que requieren ser testeadas empíricamente. Sin embargo, la naturaleza de los fenómenos en estudio plantea desafíos importantes para el logro de este objetivo. En efecto, toda vez que estos fenómenos se encuentran ya desplegados en el espacio social y están interrelacionados en complejas dinámicas, los estudios se ven restringidos a diseños de investigación observacionales. Esta restricción compromete uno de los supuestos básicos de la inferencia causal: la intercambiabilidad. Al respecto, para inferir causalidad es necesario comparar dos grupos: el que ha estado sometido al fenómeno que hipotetizamos como causa (el grupo tratamiento), y el que no ha estado sometido a él (el grupo control). El supuesto para esta comparación es que estos grupos son iguales en todo menos en la exposición a la causa; es decir, que los individuos que los componen son intercambiables entre grupos. Al no poder asignar aleatoriamente a los individuos a los distintos grupos -que es la manera más segura de garantizar el cumplimiento de este supuesto- los diseños observacionales deben incorporar procedimientos para identificar y balancear ciertas características de los individuos que podrían estar desigualmente distribuidas entre los grupos a comparar. Con estos procedimientos, los diseños observacionales aseguran un set de comparaciones válidas que a su vez permiten inferir causalidad.

En los últimos años, la importancia de clarificar los procedimientos para generar comparaciones válidas ha sido reconocida entre los investigadores de las Ciencias Sociales en el contexto de la discusión más amplia sobre el valor de la planificación de los estudios observacionales previo al trabajo de campo (Rose 2015). Siguiendo las recomendaciones de W. G. Cochran -quien en los años 60 ya reconoce la importancia de la planificación para asegurar la validez de las inferencias causales-, un plan de análisis para estudios no experimentales debiera, a lo menos, establecer el objetivo del proyecto, señalar un set de comparaciones válidas (qué se va comparar con qué) y definir la estrategia de análisis con estas comparaciones ya validadas (Cochran y Chambers 1965). Los puntos primero y tercero son condiciones sine qua non de cualquier estudio de calidad. Sin embargo, el segundo resulta ser más bien contencioso. En general, cuando la asignación aleatoria no es posible, no existe un solo modo de identificar efectos causales mediante comparaciones. En los últimos años, una perspectiva que ha ido ganando fuerza es la de generar comparaciones válidas en base al diseño de la investigación y no al modelamiento estadístico de los datos (Samii 2016). Es decir, es en el diseño de la investigación donde se toman decisiones con el objetivo de hacer comparables a los grupos tratamiento y control, intentando igualarse con un estudio experimental. Así, el énfasis está 
puesto en la composición de los grupos y no en el posible efecto del tratamiento en una variable resultado (Rosenbaum 2010). ${ }^{2}$

En este contexto, este artículo tiene por objetivo presentar una estrategia para generar comparaciones válidas. Específicamente, argumentamos que la validez de las comparaciones de un estudio observacional depende de tres elementos clave. El primero de ellos es el modelo causal, en el cual se formaliza el objetivo y las hipótesis del estudio, y que es central para identificar los supuestos bajo los cuales se puede demandar causalidad. El segundo está estrechamente relacionado con los supuestos de identificación en la medida que refiere al nivel de comprensión del proceso de auto-selección de las unidades (individuos) en las condiciones del tratamiento. Finalmente, el tercer elemento refiere al diseño de las comparaciones de un estudio, para lo cual resultan centrales las conclusiones que se puedan sacar de los dos elementos previos. En este artículo, utilizamos la técnica de Full Matching para construir comparaciones válidas. Este método es una técnica flexible que es usada para condicionar por covariables (es decir, variables observadas distintas a la variable resultado) cuando se tiene por objetivo estimar efectos causales en una situación en donde no hay asignación aleatoria. Al asumir independencia condicional o selección sobre variables observadas, un diseño de estudio observacional en base a Full Matching replica lo que sucedería si se tuviera un experimento de bloques con asignación aleatorizada dentro de cada estrato construido en base a las covariables observadas (Rosenbaum 2010). Sobre la base de estos tres elementos de la estrategia de identificación causal, se discuten algunos aspectos que pueden contribuir a la elaboración de planes de análisis para estudios observacionales.

La estrategia que proponemos es ilustrada a través del análisis de un caso de estudio: la ciudad de Chaitén y las consecuencias sociales que ha tenido la erupción del volcán del mismo nombre en el año 2008. Debido al desborde del río Blanco, a consecuencia de la erupción, se generó una división de la ciudad en dos sectores: el norte y el sur. La ciudad fue declarada inhabitable por el gobierno el mismo año, pero posteriormente, en el año 2011, el gobierno permitió la repoblación, pero solo del sector norte de la ciudad. La división norte-sur persiste hasta el día de hoy: el sector norte mantiene la condición de habitable, mientras que el sur ha sido declarado como inhabitable, aunque en él existe un número significativo de ocupaciones irregulares. En base a información cualitativa y a una encuesta aplicada en Chaitén durante 2015, este artículo utilizará la distinción entre sector norte y sector sur como tratamiento, pues esta expresa un clivaje social en torno a la distinción entre asentamiento regular e irregular. Sobre la base de este tratamiento, elaborará sobre los tres elementos arriba mencionados y evaluará su efecto en la confianza social y la cooperación de los chaiteninos, mostrando una importante autoselección de las unidades a las condiciones del tratamiento bajo estudio. Como veremos,

Variable resultado o simplemente resultado (outcome) es el término usado en el campo de inferencia causal para referirse a una variable dependiente que podría estar afectada por un tratamiento. 
dicha autoselección descarta la clasificación de este caso como un experimento aleatorizado (o experimento natural), y nos lleva a un escenario de estudio observacional en donde eventos como el de Chaitén son particularmente útiles para estudiar efectos causales, pues la población es relativamente homogénea en una serie de características, lo que hace más creíble las inferencias que se pueden hacer a partir de casos de estudio en donde el supuesto de cómo-sialeatorizado (as if random) pareciera no cumplirse.

Dado el objetivo del estudio y las características del caso mencionado, las contribuciones del artículo son las siguientes. Primero, siguiendo a Dunning (2012), los procesos sociales que determinan la asignación a las condiciones de tratados y controles son frecuentemente descuidados en estudios observacionales, particularmente aquellos que usan matching. Estas investigaciones más bien ponen el foco en el método estadístico que enfrenta los posibles sesgos en la estimación de efectos causales, por lo que el proceso de selección queda como una "caja negra". Este artículo contribuye, entonces, a llenar esta brecha investigativa al ocupar un diseño mixto que permite la sincronización del proceso de identificación del modelo causal, el diagnóstico del proceso de selección y, sobre la base de estos dos, la sincronización con el diseño de matching, que busca entregar soluciones a posibles sesgos que el diagnóstico sugiere. Dado que estos tres pasos ponen el foco en la validación de las comparaciones relevantes para el objetivo del estudio, y no en los posibles efectos de dichas comparaciones, nuestra propuesta de planificación de estudios observacionales evita algún tipo de manipulación (fishing) de los resultados, aproximándose así a uno de los objetivos de planes de análisis en estudios experimentales (Humphreys et al. 2013). Segundo, frecuentemente los estudios observacionales buscan validar las comparaciones con métodos como matching solo con el objetivo de balancear las distribuciones de las covariantes en los grupos de tratados y de controles. Sin embargo, este balance es solo un aspecto a tener en cuenta. Sugerimos criterios adicionales que serían necesarios para asegurar un buen análisis de matching, como, por ejemplo, el análisis de sensibilidad. En suma, este trabajo plantea una serie de desafíos al diseño de estudios observacionales que buscan establecer relaciones causales.

El texto está compuesto de las siguientes secciones. Primero, presentamos el caso bajo estudio. Sobre la base de información del caso, la segunda sección desarrolla los tres elementos involucrados en la estrategia de validación de comparaciones. Luego pasamos de la etapa de diseño a la de análisis. En este sentido, la tercera sección presenta los datos y el método de análisis. Las últimas secciones corresponden a los resultados de los análisis y la discusión sobre la importancia de la planificación de los estudios observaciones cuando estos están orientados a inferir causalidad, y de la relevancia del desarrollo de estrategias para generar comparaciones válidas cuando no es posible la asignación aleatoria de las unidades de análisis a los grupos tratamiento y control. 


\section{DESCRIPCIÓN DEL CASO DE CHAITÉN}

Chaitén es una ciudad-puerto ubicada en el Golfo del Corcovado, capital de la comuna homónima dependiente de la provincia de Palena, en la Región de Los Lagos de Chile. Para el año 2002 contaba con una población de 4.065 habitantes a nivel urbano (INE 2005). Entre el 2008-2009 la ciudad fue afectada por la erupción del volcán Chaitén, ubicado a 10 kilómetros al noreste de la ciudad. La erupción comenzó inesperadamente el 2 de mayo de 2008 y continuó en sus diferentes fases hasta finales del 2009 (Major y Lara 2013). Esta es considera una de las mayores erupciones de los últimos treinta años a nivel global y la más grande erupción de riolita desde comienzos del siglo $\mathrm{XX}$, con un valor en el índice de explosividad volcánica (VEI Volcánic Explosivity Index) de 4-5 (Major y Lara 2013; Carn et al. 2009).

Al momento de la erupción, el gobierno de Chile declaró la provincia de Palena "zona de catástrofe" y ordenó la evacuación total de la población de la ciudad de Chaitén y de otras localidades en el radio de 50 kilómetros en torno al volcán (Berezin 2015). En total 8.119 personas fueron evacuadas, de las cuales 5.707 provenían de la comuna de Chaitén, y fueron albergadas principalmente en las comunas de Puerto Montt, Castro y Osorno, de la región de Los Lagos, y en las comunas de Cisne y Laguna Verde, de la Región de Aysén (ONEMI 2009).

Aún cuando debido a la pronta evacuación no se registraron fatalidades humanas como consecuencia directa de la erupción, la gran cantidad de material piroclástico acumulado, que fue removido por intensas precipitaciones que afectaron la zona diez días después de la primera explosión, causó aluviones volcánicos que generaron graves daños en la ciudad (Pierson et al. 2013). En particular, el 12 de mayo del 2008 la acumulación de sedimentos en la cuenca del río Blanco (también llamado río Chaitén), que conecta con el sector sur del volcán Chaitén, produjo el desborde del río desviándolo a través de la ciudad de oriente a poniente, arrasando con aproximadamente once manzanas e inundando el resto con una mezcla de agua, madera, lodo y cenizas. Actualmente, la ciudad permanece dividida por el río, lo que ha generado la distinción entre dos sectores geográficos: el sector norte y el sector sur (Espinoza et al. 2015; Ugarte y Salgado 2014). 
Figura 1: El desastre de Chaitén: desborde del río Blanco y división de la ciudad

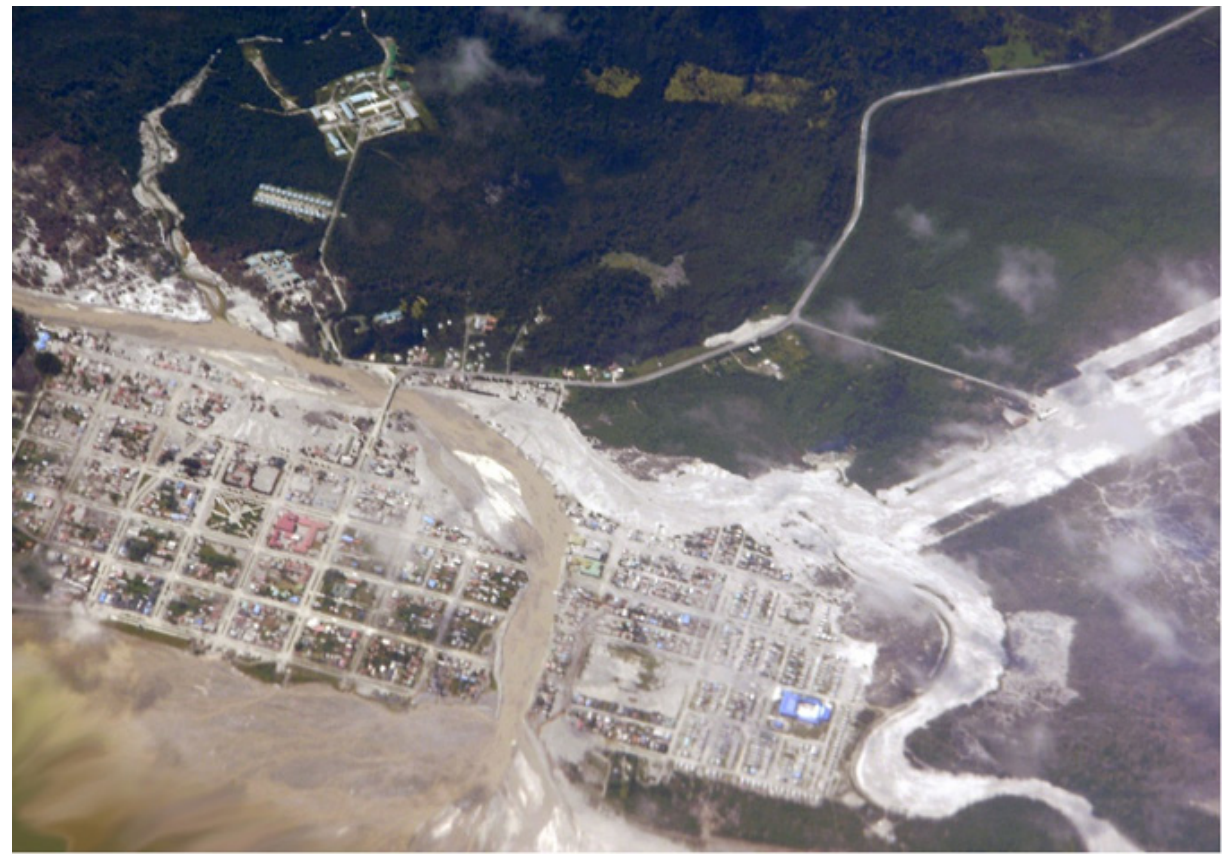

Nota: fotografía tomada días después de ocurrido el desborde del río Blanco. En la imagen, el sector norte de Chaitén está ubicado al lado izquierdo de la figura y el sector sur de la ciudad está al lado derecho.

Desde 2008 hasta 2010 se declaró inhabitable la ciudad de Chaitén y los servicios básicos como el agua y la electricidad fueron cortados. Durante la administración de Michelle Bachelet (2006-2010) se realizaron tres estudios que describían los riesgos de la ocupación de la ciudad asociados con la presencia de cenizas que cubrían su suelo y con la posibilidad de una nueva erupción, así como también evaluaban la posibilidad de reconstruir la ciudad en una localidad 10 kilómetros al norte, llamada Santa Bárbara (Observatorio de Ciudades PUC 2009). Tras la inhabilitación de Chaitén, desde el Estado se ofreció a las personas que tuvieran propiedades en la ciudad la compra de sus viviendas y se distribuyeron entre la población desplazada recursos como bonos y subsidios habitacionales para su reubicación permanente en otros lugares como Puerto Montt, Castro y Santa Bárbara. El proyecto de la reconstrucción de la ciudad de Chaitén en esta última finalmente no se llevó a cabo y fue desechado bajo la administración de Sebastián Piñera (2010-2014) por razones económicas (Ugarte y Salgado 2014).

A pesar de la prohibición de ocupación, en agosto del 2008 algunos pobladores comenzaron a regresar a Chaitén y a ocupar sus viviendas ilegalmente. No es sino hasta febrero de 2011, bajo el cambio de administración, que las autoridades resolvieron reconstruir Chaitén en el mismo lugar, habilitándose la ocupación solo del sector norte (Espinoza et al. 2015; Berezin 2015), aun cuando algunos 
estudios confirman el riesgo al que se expone la población de toda la ciudad (Lara y Calderón 2015). En la actualidad, los dos sectores de la ciudad se encuentran habitados, con una población estimada de 1732 habitantes; 1180 en el sector norte y 552 personas en ocupaciones irregulares en el sector sur (Ministerio del Interior y Seguridad Publica de Chile 2014), lo que representa el $42,6 \%$ de la población previa a la erupción.

\section{ESTRATEGIA PARA LA VALIDACIÓN DE LAS COMPARACIONES EN ESTUDIOS OBSERVACIONALES}

En el escenario más simple, para establecer relaciones causales se requiere comparar dos grupos: el grupo tratamiento y el grupo control. El grupo tratamiento está constituido por los individuos expuestos al fenómeno que queremos establecer como causa. El grupo control, por el contrario, agrupa a los individuos que no están sujetos al fenómeno en cuestión. Para que esta comparación sea válida, las covariables observadas -que no son sino otras variables medidas en el estudio y que caracterizan a los individuos-deben estar balanceadas en ambos grupos; por ejemplo, los grupos no se diferencian en el promedio de edad de sus individuos. En los estudios observacionales, al no haber una asignación aleatoria de los individuos a los distintos grupos, este balance se ve comprometido -puede ser el caso que uno de los grupos tenga un promedio de edad mayor al otro-. Frente a este escenario, los estudios observacionales demandan una estrategia para validar las comparaciones. Tal como enunciamos en la introducción, esta estrategia involucra tres pasos. El primero es definir de manera clara y consistente el modelo causal que subyace a las hipótesis del estudio. Este modelo formaliza dichas hipótesis y está compuesto de variables y de los supuestos necesarios para identificar la asociación entre las variables en cuestión como una relación causal. El segundo paso es examinar el proceso de selección de los individuos a los distintos grupos en base a información empírica, de modo de evaluar la consistencia de las variables y la validez de los supuestos definidos por el modelo. El tercer paso es diseñar o utilizar una técnica para balancear las covariables en ambos grupos. A continuación expondremos en detalle los pasos descritos.

\section{DEFINICIÓN DEL MODELO CAUSAL: TRATAMIENTO Y EFECTOS}

Un aspecto clave del diseño de estudios observacionales es definir el modelo causal, que implica como primer paso identificar claramente el tratamiento y los efectos esperados en la variable de resultado; es decir, las variables independiente y dependiente de interés. Más aun, se puede argumentar que la definición del tratamiento en estudios observacionales resulta ser más importante que la clarificación del resultado, pues, por definición, este no está sujeto a la manipulación del investigador y, por lo tanto, su consistencia 
es debatible. Al respecto, un ejemplo estándar es un tratamiento asociado con límites geográficos. En este caso es difícil señalar con claridad cuál es exactamente la intervención, pues el territorio puede reflejar una combinación de varios tratamientos (Keele y Titiunik 2016). Así, siguiendo a Dunning (2012), la clave para tratamientos de este tipo está en identificar qué aspecto específico del grupo de tratados, como opuesto al de controles, puede causar diferencias en alguna variable de interés.

A modo de ilustración, este artículo identifica la división territorial norte/sur, producida como consecuencia de la erupción del volcán Chaitén, como el aspecto que puede causar diferencias entre ambos grupos. En términos sustantivos, el norte y el sur se diferencian en su estatus de asentamiento regular/irregular, pues, tal como vimos en la sección anterior, es solo el sector norte el que está habilitado formalmente por el Estado para ser habitado. En términos específicos, la irregularidad de un asentamiento refiere a la violación tanto de los derechos de propiedad de los suelos ocupados como de las regulaciones urbanísticas y de disposiciones tributarias (Fernandes 2011). En este sentido, el tratamiento bajo análisis en el presente estudio es el habitar o residir por parte de los individuos en un asentamiento irregular. También, a modo de ilustración, desde un punto de vista sociológico y psicológico, se podría hipotetizar que las condiciones de irregularidad tienen un efecto en fenómenos como la cooperación y la confianza. De esto modo, hemos planteado una hipótesis general y definido las variables del modelo causal que nos permiten precisar la comparación que será realizada en el estudio.

\section{LA AUTOSELECCIÓN Y EL SUPUESTO DE INTERCAMBIABILIDAD}

Una vez identificados el tratamiento y los resultados, los pasos siguientes son definir los supuestos que señalan bajo qué condiciones ambas variables están relacionadas de un modo causal y examinar empíricamente el proceso de selección. Siguiendo la literatura de inferencia causal (Imbens y Rubin 2015; Hernan y Robins 2006), el supuesto básico es el de intercambiabilidad o independencia. ${ }^{3}$ Básicamente, este supuesto significa que los grupos de tratados y controles son intercambiables en todas las características observadas y no observadas relevantes para el proceso de selección. En otras palabras, y usando los términos contemporáneos de inferencia causal, los tratados son contrafactuales válidos de los controles y viceversa. En estudios experimentales, este supuesto está garantizado por la selección aleatoria de los individuos en las distintas condiciones del tratamiento. En estudios observacionales, tal no es el caso y por esta razón es que puede ser necesario examinar empíricamente el proceso de selección de modo de entender qué factores alejan al estudio

Ciertamente, argumentos sustantivos y/o teóricos son centrales para definir una relación causal. En este artículo, el foco está puesto más bien en los supuestos metodológicos para identificar causalidad. 
observacional de un diseño aleatorizado. Un estudio observacional puede demandar causalidad bajo el supuesto de independencia condicional, lo que significa que los tratados y controles son intercambiables si los condicionamos por factores observados relevantes para el proceso de selección y asumimos independencia del tratamiento respecto de factores no observados. ${ }^{4}$

Para la identificación de dichos factores, resulta clave entender que las ciencias sociales tienen la particularidad de que las unidades bajo estudio son, por lo general, individuos que actúan bajo incentivos y restricciones. En consecuencia, el estudio del proceso de selección de actores a las condiciones de un tratamiento debe tomar en cuenta estas características. En efecto, en términos conductuales, la intercambiabilidad implica que los sujetos bajo estudio no eligen si se ubican en alguna de las condiciones experimentales. Para que esto suceda, tal como afirma Dunning (2012), los actores deben carecer de información, incentivos o capacidades que les permitan influenciar la asignación del tratamiento. Se podría decir que, si se dan estas condiciones, un estudio estaría muy próximo a un diseño experimental. Si extrapolamos este razonamiento a la comprensión de estudios observacionales, queda en evidencia que, desde un punto de vista conductual, la no aleatoriedad que define a este tipo de diseño refiere a que los sujetos, en base a la información o incentivos de que disponen, pueden decidir en qué condición del tratamiento prefieren estar. En este sentido, el proceso de selección en los estudios observacionales puede ser entendido como autoselección, en tanto es un proceso social sujeto a la decisión de individuos.

Evidentemente, la autoselección de los sujetos en estudios no experimentales puede estar asociada con una serie de factores. Pues bien, el examen del proceso de autoselección justamente nos puede ayudar a identificar qué variables están determinando las decisiones de los agentes. La importancia de este paso refiere a que un estudio observacional puede garantizar comparaciones válidas entre tratados y controles solo si incluye estas variables en su diseño. En otras palabras, y dicho de un modo más técnico, estas variables pueden ser utilizadas para el balanceo de las covariables en los grupos de comparación mediante, por ejemplo, el Full Matching de modo de que el estudio se aproxime a un diseño experimental.

¿Hay evidencia de autoselección de los sujetos a las condiciones de tratamiento en el caso de Chaitén? Dicho de otro modo, ¿puede decirse que la ubicación de los habitantes de Chaitén al lado norte o sur de río Blanco es aleatoria? La evidencia cualitativa recolectada sugiere que hay una importante autoselección de las personas en las condiciones del tratamiento bajo estudio, lo que indica que la intervención no corresponde ni a un experimento aleatorizado ni a un experimento natural. Específicamente, los datos cualitativos revelan dos grupos de factores que serían centrales para entender las determinantes de la ubicación

En el apéndice, presentamos una formalización de los efectos causales y del supuesto de intercambiabilidad e independencia condicional, con el fin de exponer claramente en qué consisten ambos aspectos clave para el estudio de inferencia causal. 
de los individuos, ya sea en el sector norte o en el sur. El primero refiere a la historia residencial de las personas: la evidencia cualitativa que recolectamos indica que el haber habitado Chaitén antes del desastre generó incentivos para rehabitarla después de este. Como se rescata de la literatura y las entrevistas realizadas, estos incentivos se relacionan fuertemente con aspectos psicológicos vinculados con dimensiones identitarias que relacionan a la persona con el lugar habitado. Haber nacido en el lugar influye en el apego que se tiene hacia este (Hernandez et al. 2007), lo que se reconoce entre los entrevistados como un incentivo para regresar. El siguiente relato ilustra lo anterior: "Bueno... yo nací aquí, me crié aquí, aqui quiero forjar mi futuro y aqui quiero también quedarme para siempre. Porque está también ese arraigo a la tierra". El haber nacido o habitado Chaitén, entonces, podrían motivar a las personas a volver a ocupar los antiguos lugares de residencia, ya sea en el sector norte o en el sur.

El segundo grupo de factores de autoselección refiere a las condiciones materiales de existencia de los habitantes de la ciudad. En efecto, previo a la erupción del volcán, ya existía una división social en Chaitén en torno al sector norte y al sector sur, que descansaba fuertemente en el estatus socioeconómico de los habitantes. Como nos cuenta un habitante del sector sur: "Chaitén, pa' nosotros antiguamente habian dos sectores: era sector sur y sector norte, estaba dividido por el deporte y esas cosas; características de Chaitén era, eso era bien marcado, el tema de lo del sector norte, sector sur. Nosotros vivíamos en una parte que le pusieron 'el barrio de los chicos pobres', no sé si lo habias escuchado pero... era que la gente como que estaba en... del hospital, o de la escuela básica, del hospital hasta una parte, una calle Lautaro era como que estaban en el centro ello, después estaba el sector norte y estábamos nosotros en el sector sur".

Las condiciones posdesastre indican que esta distinción socioeconómica ha vuelto a emerger pero con nuevos matices. Junto con la dimensión simbólica del clivaje pobre/no pobre, el desastre ha añadido claras condiciones de deprivación material, como ausencia de servicios básicos en el sur, y la distinción entre un asentamiento formal y otro informal que puede reforzar la distinción en base a estatus socioeconómico. Al respecto, la evidencia que recolectamos de fuentes secundarias indica que: "un grupo de familias, no más de cien personas, volvió para instalarse en la parte sur del pueblo, dividido en dos partes por el río, sector este último sin red de agua potable, alcantarillado y red eléctrica. (...) Otro factor que se ha escapado de la gestión pública y donde la propia gente ha tomado su camino, en contraposición al Estado, ha sido la instalación de al menos 120 familias en el lado sur del Chaitén, a contrapelo de las decisiones del gobierno central y en acuerdo con el gobierno local" (Tapia 2015: 151, 155). En comparación con el sector norte, la evidencia cualitativa, por lo tanto, indica claramente que los habitantes del sur de Chaitén están caracterizados por un estatus socioeconómico más bajo.

Finalmente, un elemento adicional que hay que considerar para la autoselección es la endogeneidad de los factores identificados. Tal como señala Rosenbaum (1984), el uso de covariables endógenas -es decir, variables susceptibles de ser afectadas por el tratamiento y/o por el resultado- en la estimación de los efectos 
puede introducir sesgos postratamiento. La forma más simple de evitar este problema es usar covariables medidas antes de la ocurrencia del tratamiento. Muchos estudios observacionales, sin embargo, no tienen información de este tipo. En tal situación, es recomendable discutir si los factores relevantes para la autoselección son susceptibles de ser fuertemente endógenos. Por ejemplo, es claro que la historia residencial está menos afecta al hecho de vivir en el norte o el sur que el estatus socioeconómico.

En suma, la evidencia presentada sobre el proceso de autoselección sugiere que los factores relacionados con la historia residencial y el estatus socioeconómico podrían producir desbalances entre el grupo de control y el de tratamiento; por lo tanto, son candidatos para ser incluidos en el procedimiento de Full Matching. Así, estos factores revelan la no aleatoriedad de la asignación de las unidades a los sectores norte y sur. A continuación exponemos cómo el método de Full Matching puede lograr balancear estas covariables, de modo que un estudio observacional se acerque a un experimento aleatorizado.

\section{TÉCNICA PARA BALANCEAR COVARIABLES: FULL MATCHING Y ANÁLISIS DE SENSIBILIDAD}

Rosenbaum (1991) introdujo la técnica de Full Matching en el campo de estudios observacionales y Hansen (2004), posteriormente, explicó e ilustró el funcionamiento del método. ${ }^{5}$ Sobre la base de un set de covariables definidas previamente, Full Maching construye bloques conformados por tratados y controles de modo tal que ambos grupos estén balanceados en las distribuciones de las covariables. En consecuencia, construye un diseño de bloques, en donde los tratados y los controles dentro de cada bloque son intercambiables respecto de las covariantes observadas utilizadas en la generación de estos. En comparación con modelos de regresión, Full Matching es un tipo de estimador semiparámetrico que intenta evitar hacer supuestos sobre la forma funcional de la asociación entre las variables, e identifica una zona de interpolación en donde tratados y controles son comparables en las covariables observadas. Dicho de otro modo, Full Matching empareja peras con peras -las que se distinguen solo en que una es tratada y la otra es control- y no peras con manzanas, como puede ser el caso de un modelo de regresión (Ho et al. 2007). El diseño de bloques generado por el matching corresponderá a un experimento aleatorizado bajo el supuesto de selección sobre covariables observadas. Como ilustramos más abajo, el análisis de sensibilidad ha sido propuesto para evaluar este supuesto con diseños observacionales. A diferencia de otros tipos de matching (conocidos con el nombre de Greedy Matching) que están disponibles en softwares de uso habitual en ciencias sociales, como SPSS o Stata, Full Matching optimiza el balance. Además, a diferencia de los que forman parejas de tratados y controles (conocidos como Pair Matching), los bloques generados por Full Matching 
pueden estar conformados por un número variable de controles y de tratados. De este modo, se evita perder casos.

Para lograr que un matching contribuya a una identificación válida de efectos causales, tres decisiones del diseño de los matchs son centrales. Primero, el diseño debe contemplar de qué modo serán consideradas las covariables para la formación de los bloques, teniendo en cuenta que formar grupos de tratados y controles es difícil cuando el número de covariables aumenta. Una manera de solucionar este problema es usar lo que se conoce como propensity score, que es la probabilidad condicional de asignación al tratamiento dadas las covariables observadas. Siguiendo a Rosenbaum y Rubin (1983), si no condicionamos directamente por las covariables sino por el propensity score podemos lograr tanto balance de las covariables entre los grupos de tratamiento y de control como independencia del tratamiento respecto de los resultados. Dichos supuestos dependen de que el propensity score esté correctamente especificado (Ho et al. 2007).

Una segunda decisión de diseño refiere a que el número de tratados y controles dentro de cada bloque generado por el procedimiento de Full Matching sea similar; por ejemplo, tener bloques con 50\% de tratados y 50\% de controles. Si la razón de tratados y controles dentro de cada bloque está muy desbalanceada, dicho problema puede introducir sesgo en las estimaciones (Stuart y Green 2008). Tal como sugiere Hansen (2004), Full Matching permite solucionar este problema a través de la imposición de restricciones al número de tratados y controles que habrá dentro de cada bloque. Por ejemplo, definir de antemano el número mínimo y el número máximo de controles dentro de cada bloque. Finalmente, la tercera decisión refiere al modo que el estudio observacional considera el rol de variables no observadas en su diseño. Un aspecto clave del supuesto de independencia condicional sobre el que trabaja el matching es que los tratados y controles son intercambiables solo bajo el condicionamiento de las variables observadas relevantes. Sin embargo, puede suceder el caso de que alguna covariable, la cual afecte tanto al tratamiento como al resultado, no sea considerada en el procedimiento de matching. En dicha situación, los tratados y los controles no serían comparables y, por lo tanto, no podríamos identificar el efecto causal en cuestión sin sesgo. Solo el procedimiento de asignación aleatorizada garantiza el balance tanto de las covariables observadas como de las no observadas.

Frente al escenario de la ausencia de una covariable relevante, los estudios observacionales contemplan lo que se conoce como análisis de sensibilidad. Rosenbaum (2010) afirma que un análisis de sensibilidad en un estudio observacional se pregunta en qué medida sus conclusiones cambian si las unidades que se ven comparables gracias al matching fueran más bien diferentes. Uno de los enfoques sobre análisis de sensibilidad más usados en la literatura es el del propio Rosenbaum (2015). Este está interesado en encontrar criterios límite, conocidos como Rosenbaum's bounds, de la asociación entre la covariable no observada y el tratamiento y/o entre esta covariable y la variable resultado, 
que podrían hacer al efecto causal identificado no significativo desde un punto de vista estadístico. Este enfoque ha sido creado para funcionar especialmente con matching de pares o Full Matching y está compuesto por tres tipos de análisis de sensibilidad. Uno de ellos es el análisis de sensibilidad primario, en donde se hace variar a modo de simulación la asociación entre el tratamiento y la variable no observada, asumiendo que esta última predice perfectamente la variable dependiente. Por ejemplo, si $r$ denota la fuerza de dicha asociación, el objetivo de este tipo de análisis es encontrar el valor de $r$ en donde el efecto del tratamiento pierde significación estadística. En contraste, el análisis de sensibilidad dual varía la asociación entre la variable no observada y la variable dependiente, dejando que la covariable no observada prediga perfectamente el tratamiento. En este caso, el límite crítico es designado con A. Finalmente, el análisis de sensibilidad simultaneo identifica la sensibilidad del efecto encontrado en distintas combinaciones de $r$ y A. ${ }^{6}$ En las siguientes secciones, ilustramos cómo las tres decisiones relevantes para el diseño de los matchs fueron tomadas para analizar los datos sobre el caso de Chaitén.

\section{VII.DATOS Y MÉTODO DE ANÁLISIS}

\section{Diseño muestral y trabajo de campo}

Los análisis presentados en este artículo están basados en la Encuesta de Decisión de Ocupación de Lugares Expuestos a Amenazas Naturales, que fue diseñada y aplicada en el marco del proyecto en curso Habitar y Re-habitar una Comunidad afectada por un Desastre Natural: el Caso de las Erupciones del Volcán Chaitén en el 2008, desarrollado con apoyo del proyecto FONDAP Centro Nacional de Investigación para la Gestión Integrada de Desastres Naturales (CIGIDEN). La población objetivo del estudio fueron personas de 18 años o más residentes de la ciudad de Chaitén, región de Los lagos de Chile. El tamaño estimado de esta población es de 1,732 habitantes en total, 1,180 en el Sector Norte y 552 en el Sector Sur (Ministerio del Interior y Seguridad Publica de Chile 2014).

El trabajo de campo en zonas de desastre representa una serie de desafíos. Uno de ellos es la ausencia de marco muestral a partir del cual diseñar una muestra aleatoria. Chaitén no es una excepción a esta situación. Para paliar la carencia de un marco muestral, el proyecto recién mencionado contempló la realización de un empadronamiento general de la ciudad durante octubre de 2015 para identificar las viviendas ocupadas. Este arrojó un total de 566 viviendas, 337 en el sector norte y 196 en el sector sur, y 33 en el camino Aeródromo, hacia el este de la ciudad. Estas últimas se incorporaron al total de viviendas del sector Norte, ya que son ocupaciones regulares, contabilizando el sector norte un total de 370.

Para un desarrollo más formal de este tipo de análisis de sensibilidad en ciencias políticas, ver Budziak y Lempert (2015). 
Del total de viviendas se excluyeron aquellas de uso comercial o administrativo, dando un total de 491 viviendas ocupadas para uso habitacional, 271 en el sector norte y 220 en el sector sur.

Respecto del diseño muestral, el proyecto se planteó como objetivo primario levantar datos de la más alta calidad. Para ello se diseñó una muestra estratificada por conglomerado bi-etápico. Los estratos se definieron geográficamente en los dos sectores de la ciudad de Chaitén, sector norte y sector sur, con un tamaño muestral objetivo de 251 entrevistas, para obtener un error muestral de 5,8 puntos, suponiendo varianza máxima, un nivel de confianza del 95\% y una población de 1.732 personas en Chaitén. En la primera etapa se seleccionaron aleatoriamente viviendas particulares ocupadas en cada uno de los estratos (Unidad de Muestreo Primaria - UMP), de forma proporcional a su tamaño, utilizando como marco muestral el empadronamiento general de la ciudad. Un total de 314 UMP fueron seleccionadas, 193 en el Sector Norte y 121 en el Sector Sur, asumiendo una tasa de sobredimensión de 20\%. En la segunda etapa se listó a todas las personas residentes habituales de cada UMP con edad de 18 años o más, entre los que se seleccionó una persona (Unidad de Muestreo Secundaria - UMS) aleatoriamente mediante Tabla Kish. En base a este diseño muestral, se aplicó un total de 182 entrevistas durante noviembre y diciembre de 2015, 39 fueron inválidas, considerándose casos neutrales no entrevistados, dando una muestra neta de 274 entrevistas, que representan el $87 \%$ de la muestra bruta. La tasa de respuesta general fue de 0,66 , siendo menor en el estrato del sector norte, con una tasa del 0,62 en comparación con la tasa de 0,72 en el sector sur. Dependiendo de la presencia de casos perdidos en las variables consideradas, las muestras utilizadas en los análisis varían entre 153 a 168 casos.

\section{Mediciones: tratamiento, variable resultado y covariables}

En este estudio, la variable resultado refiere a las nociones de confianza y cooperación, que son operacionalizadas por un set de indicadores de dichos conceptos que son ampliamente ocupados en la literatura. ${ }^{7}$ El tratamiento está medido por una variable dummy en donde 1 designa a encuestados que habitan el sector sur de Chaitén (tratados) y 0 denota a habitantes del sector norte (controles). Como ya se dijo, esta medición captura a nivel de las personas la distinción entre habitar un asentamiento irregular y habitar un asentamiento sujeto a regulaciones formales del Estado. Si bien esta medición puede aparecer como algo general, análisis de indicadores de presencia del Estado en el territorio confirman que el sector norte y sur se distinguen según nivel de regulación estatal. ${ }^{8}$

Para un reciente resumen de la literatura sobre confianza y su medición, ver Abascal y Baldassarri (2015). Nuestro estudio incorporó una serie de mediciones de estatalidad desarrolladas por Luna y Soifer (2015). Los indicadores refieren a la regulación tanto de los derechos de propiedad como del territorio en términos de la capacidad de la policía de llegar a los hogares de los entrevistados. Los análisis revelan que la regulación de ambas dimensiones es menor en el sur de Chaitén. Así encontramos que la policía significativamente 
Hemos medido confianza con tres indicadores. El primero es confianza generalizada y ha sido operacionalizada por la pregunta estándar de encuesta: "Hablando en general, ¿diría usted que se puede confiar en la mayoría de las personas o que uno nunca es lo suficientemente cuidadoso en el trato con los demás?". En base a esta pregunta, se construyó una variable dummy en donde 1 indica confía y 0 no confía. Los siguientes dos constructos de confianza refieren a confianza intra e intergrupal (in-group / out-group trust). Ambas mediciones fueron construidas en base a preguntas de confianza con escalas de respuesta de cuatro puntos que van desde "confía completamente" a "confía nada". Siguiendo a Delhey et al. (2011), la confianza intragrupal refiere a la confianza en grupos de cercanos y es operacionalizada por el nivel de confianza en tres grupos, a saber, familia, vecinos y conocidos personales. Las respuestas a cada uno de estos tres grupos fueron agrupadas en un índice sumativo que va de 0 (no confianza) a 1 (confianza). Por su parte, la confianza extragrupal refiere confianza en grupos de extraños y fue medida por el nivel de confianza que el encuestado declara por personas que conoce por primera vez, por personas de otra religión y por gente de otra nacionalidad. Del mismo modo que la confianza intragrupal, también en este caso se construyó un índice sumativo. Finalmente, en adición a nuestras mediciones de confianza, consideramos un indicador de cooperación que es construido a través de la siguiente pregunta: "Imagínese que estas diez personas son habitantes de Chaitén. Desde la municipalidad le piden a cada uno de los habitantes de Chaitén que ocupen menos bencina en caso de una nueva erupción que aísle la ciudad. [Se le muestra al encuestado una tarjeta con 10 figuras de personas]. De estas diez personas, ¿cuántos cree usted que cooperarían con la instrucción de la municipalidad?". La pregunta busca capturar la percepción de cooperación en la comunidad en cuestión. Idealmente la pregunta debiera considerar un bien público (por ejemplo, educación). Sin embargo, nos inclinamos por bencina pues es el recurso que fue frecuentemente mencionado en las entrevistas cualitativas cuando se preguntó por la evacuación.

Las covariables fueron incluidas en la estimación de matching a través de la estimación del propensity score respectivo. Para esto utilizamos dos tipos de factores. El primer grupo refiere a variables sobre la historia residencial de los encuestados. Tal como discutimos en la sección sobre el proceso de autoselección, hay buenas razones para pensar que las personas que estuvieron en Chaitén durante las erupciones volcánicas de 2008, y/o que han nacido o vivido en Chaitén, tienen incentivos para volver al lugar donde previamente habitaron. En consecuencia, nuestros análisis variables incluyeron dummies que capturan la historia residencial de los entrevistados en términos de si el encuestado estuvo en Chaitén durante el 2008, si nació en Chaitén y si vivió en esta ciudad antes del desastre. Además, incluimos una variable dummy para la condición de si 
llegó antes o después de 2011, pues nuestras entrevistas sugieren que la mayoría de los nuevos ocupantes del sur llegaron a Chaitén después de esta fecha.

El segundo tipo de covariables refiere a factores sociodemográficos. Dentro de estos, destaca el estatus socioeconómico, pues, tal como ya se dijo, personas con un bajo estatus socioeconómico se estarían autoseleccionando en nuestra condición de tratamiento (vivir en el sur). Específicamente, consideramos el estatus socioeconómico a través de mediciones del ingreso del hogar y del nivel educacional del encuestado, que fue considerado como una variable binaria con 1 para educación media o más y 0 para básica o menos. Respecto del ingreso, se creó una variable categórica en base a cuartiles. Dado el bajo número de casos en algunos cuartiles, se crearon tres grupos. Estos son el primer cuartil, que va desde el valor mínimo al 25\% de la distribución del ingreso; el segundo grupo incluye desde el segundo hasta el cuarto cuartil, y un grupo que identifica los encuestados que no declararon sus ingresos. Siguiendo el enfoque de Rosenbaum (2010), si se incluye esta última categoría como una covariable binaria en la estimación del matching, también se puede lograr balance sobre esta, evitándose sesgos en la estimación de efectos causales asociados con casos perdidos. ${ }^{9}$ Con el fin de balancear otras variables sociodemográficas, incluimos indicadores de características del encuestado asociadas al sexo, la edad, la etnia, el estatus laboral, la religión, el número de personas en el hogar y el estatus civil.

En adición a la relevancia sustantiva de las covariables, tal como ya se dijo, un criterio adicional a tener en cuenta en la selección de las variables es si estas fueron medidas antes o después de ocurrido el tratamiento. La selección de las covariables recién expuestas trata de evitar la inclusión de variables que pudieran estar fuertemente afectadas por el tratamiento, por ejemplo, apego al lugar o valoraciones de las consecuencias asociadas con habitar Chaitén. Lamentablemente, dado que no se dispone de mediciones previas a la ocurrencia del desastre, es importante mencionar que no podemos descartar del todo la presencia de algún tipo de sesgo postratamiento en nuestras estimaciones. En el caso del ingreso, por ejemplo, puede suceder que vivir en el asentamiento irregular del sur tenga algún costo económico para los residentes en términos de generar menores oportunidades de empleo. Cabe destacar, sin embargo, que la evidencia que recolectamos en el trabajo de campo no sugiere problemas de este tipo. De todos modos, en las conclusiones de este artículo discutimos con más detalle problemas de sesgo postratamiento y planteamos implicancias para una investigación futura.

9 Cabe destacar que si los ingresos no declarados son un efecto del tratamiento, el sesgo asociado a datos perdidos puede aun persistir. 


\section{CONSTRUCCIÓN DE FULL MATCHING Y ANÁLISIS DE VARIABLES RESULTADO}

El diseño de Full Matching ha sido determinado por dos decisiones. La primera refiere al tratamiento de las covariables dentro del diseño. Para construir los bloques generados por Full Matching, utilizamos propensity score como medida de distancia entre las unidades. Un tamaño muestral limitado y la presencia de pocos casos en algunas de las covariables pueden generar problemas en el estimativo del propensity score en base a una regresión logística. Como solución, estimamos nuestra medida de distancia utilizando una regresión de Lasso, que genera estimaciones más estables en escenarios de muestras pequeñas y de variables con alto nivel de correlación entre sí (James et al. 2013). ${ }^{10}$ La segunda decisión de construcción del Full Matching refiere a los criterios que se buscó maximizar. Al respecto, el diseño que utilizamos tuvo por objetivo maximizar el balance de las covariables, el tamaño muestral y el balance del número de tratados y controles dentro de cada bloque. Para ello, utilizamos Full Matching con restricciones, que refieren al número mínimo y máximo de controles dentro de cada bloque. Luego de una serie de iteraciones, utilizamos como restricciones una razón de 1 control por 10 tratados como mínimo y 10 controles por 1 tratado como máximo. ${ }^{11}$

En relación con el análisis de las variables resultado, el efecto causal del tratamiento en dichas variables fue identificado en base al estimador de efecto causal promedio para diseños con bloques, utilizando ponderadores (inverse probability weights). Este efecto causal es un promedio ponderado de los efectos causales de cada bloque, siendo estos últimos simplemente la diferencia de medias entre tratados y controles (Gerber y Green 2012). Los test estadísticos fueron estimados en base a inferencia aleatorizada. Este tipo de inferencia utiliza la misma aleatorización como fuente de variabilidad, con lo cual se evita la dependencia de los test estadísticos de la teoría estadística estándar basada en el supuesto de normalidad. Dicho procedimiento es especialmente útil para analizar muestras pequeñas. ${ }^{12}$ Cabe destacar que el hecho de que se disponga de efectos causales para cada bloque permite explorar la heterogeneidad causal del efecto promedio encontrado en el sentido de que tal vez los individuos pertenecientes a bloques distintos reaccionen de manera diversa al tratamiento bajo estudio, y el efecto promedio puede ocultar tal diversidad. Nosotros explotamos la estructura de bloques generados por el Full Matching, ilustrando la heterogeneidad causal para efectos promedios que resulten con bajo nivel de incerteza estadística. Finalmente, la sensibilidad de las estimaciones a variables

10 En la estimación del propensity score, las variables independientes fueron ingresadas aditivamente. También se evaluaron modelos alternativos que incluyen polinomios y efectos de interacción, sin encontrarse diferencias significativas ni para el balance de las covariables ni para los efectos causales presentados. Dado este patrón, la sección de resultados solo muestra los análisis que utilizan el propensity score generado en base a una función aditiva de covariables.

11 Además se estimaron Full Matchings sin restricciones y matching con caliper sobre el propensity score. El Full Matching con restricciones supera a ambas estrategias en los tres criterios mencionados.

12 Para un explicación de inferencia basada en aleatorización, ver Keele et al. (2012). 
no observadas, que pudieran confundir el efecto estimado, fue evaluada utilizando el análisis de sensibilidad simultaneo ya descrito.

\section{RESULTADOS}

\section{Balance}

El balance de las covariables antes y después del matching es mostrado en la Tabla 1. Evaluamos el balance logrado en base a un test ómnibus y a la inspección de diferencias estandarizadas y de las medias entre tratados y controles para cada covariable. ${ }^{13} \mathrm{El}$ test ómnibus considera todas las covariables en su conjunto y señala cuánto se acerca el diseño logrado por el matching a un experimento aleatorizado de bloques, el cual está caracterizado por un valor $p$ de 1 (Hansen y Bowers 2008). Si dicho valor $p$ es muy bajo (por ejemplo, menor de 0.05), habría fuerte evidencia de desbalances en los resultados del matching. En nuestro caso, el test ómnibus indica un fuerte desbalance en los datos pre-matching, con un valor $p$ menor de 0.001 ( $x^{2}$ de 73.8 con 16 grados de libertad). Por el contrario, el test ómnibus con los datos pos-matching genera un valor $p$ de $0.75\left(\mathrm{x}^{2}\right.$ de 11.9 con 16 grados de libertad), lo cual sugiere que nuestro Full Matching con restricciones logra excelentes resultados de balance en las variables observadas. 
Tabla 1: Balance de covariables: antes y después del matching

\begin{tabular}{ccccccc} 
& \multicolumn{3}{c}{ Pre-matching } & \multicolumn{3}{c}{ Post-matching } \\
\cline { 2 - 7 } & Norte & Sur & Dif.std. & Norte & Sur & $\begin{array}{c}\text { Dif. } \\
\text { std }\end{array}$ \\
\hline Mujer & 0,50 & 0,68 & 0,37 & 0,56 & 0,61 & 0,10 \\
Edad & 43,15 & 41,24 & $-0,15$ & 43,28 & 42,05 & $-0,09$ \\
Etnia & 0,20 & 0,25 & 0,13 & 0,28 & 0,26 & $-0,04$ \\
Estatus laboral & 0,20 & 0,22 & 0,06 & 0,21 & 0,16 & $-0,13$ \\
Religión & 0,36 & 0,40 & 0,08 & 0,49 & 0,47 & $-0,03$ \\
Casado & 2,95 & 2,72 & $-0,16$ & 2,82 & 2,79 & $-0,02$ \\
Otro & 0,46 & 0,35 & $-0,23$ & 0,40 & 0,41 & 0,02 \\
Soltero & 0,10 & 0,14 & 0,11 & 0,15 & 0,12 & $-0,07$ \\
Primaria & 0,44 & 0,57 & 0,26 & 0,45 & 0,50 & 0,09 \\
Secundaria o mas & 0,20 & 0,49 & 0,64 & 0,42 & 0,38 & $-0,09$ \\
Ingreso: missing & 0,80 & 0,51 & $-0,64$ & 0,58 & 0,62 & 0,09 \\
Ingreso: Q1 & 0,03 & 0,46 & 1,21 & 0,11 & 0,15 & 0,13 \\
Ingreso: Q2-Q3 & 0,15 & 0,25 & 0,27 & 0,30 & 0,23 & $-0,19$ \\
Estuvo el 2008 (No=1) & 0,82 & 0,29 & $-1,27$ & 0,59 & 0,62 & 0,07 \\
Nacer en Chaitén (No=1) & 0,39 & 0,28 & $-0,23$ & 0,33 & 0,30 & $-0,06$ \\
Vivión en Chaitén antes del & 0,64 & 0,53 & $-0,22$ & 0,49 & 0,49 & $-0,01$ \\
2008 (No=1) & 0,29 & 0,21 & $-0,19$ & 0,21 & 0,22 & 0,04 \\
Llegado el 2011 o después & 0,72 & 0,57 & $-0,32$ & 0,68 & 0,67 & $-0,01$ \\
\hline
\end{tabular}

Fuente: Encuesta de Decisión de Ocupación de Lugares Expuestos a Amenazas Naturales.

Nota: todas las medias son proporciones, salvo en el caso de la edad y el número de personas en el hogar. Luego de eliminación de casos perdidos (listwise), el tamaño muestral pre y pos matching utilizado es 168.

Las diferencias estandarizadas complementan al test ómnibus en la medida que ellas permiten evaluar el balance para cada covariable por separado. Diferencias menores de 0,25 en términos absolutos son habitualmente consideradas como un balance aceptable (Stuart y Green 2008). Los resultados de la Tabla 1 en la condición pre-matching indican que el balance sería aceptable para la mayoría de las covariables salvo el género, el indicador de si el encuestado llegó en 2011 o después a Chaitén y, sobre todo, las variables de estatus socioeconómico (ingreso y nivel educacional). Como ilustración, mientras el $82 \%$ de las personas que habitan en el sector norte declaran un ingreso del hogar que está por sobre el primer cuartil, solo el 30\% del sector sur se ubica en este grupo de ingreso. Ahora bien, cuando vemos las estimaciones pos-matching, el balance mejora notablemente. Todas las covariables presentan diferencias estandarizadas menores de 0,20. Respecto de la ilustración mencionada, tenemos que en el pos- 
matching solo hay una diferencia de 3 puntos porcentuales entre los del sector norte y del sector sur respecto al grupo de mayor ingreso (59\% vs. 62\%).

\section{Estimación de efectos}

La Figura 2 muestra los efectos causales promedio estimados en base a los datos procesados por Full Matching. La línea vertical roja indica un efecto de cero. Los puntos negros denotan los efectos estimados y las líneas en torno a los puntos representan los intervalos de confianza al 95\%. ${ }^{14}$ Tenemos un efecto estadísticamente significativo distinto de cero si los intervalos no cruzan la línea roja. Tal como podemos ver en la figura, los resultados indican tanto efectos significativos como ausencia de diferencias entre tratados y controles. Respecto de esto último, no observamos efectos significativos de residir en un asentamiento irregular en la confianza social, tanto generalizada como intra y extragrupal. El panorama, en cambio, es distinto para la cooperación. Los resultados indican que solo para esta variable existe un efecto del tratamiento estadísticamente significativo, con un intervalo de confianza al 95\% de [0,06, $3,14]$. El efecto estimado es de 1,61 y quiere decir que, cuando se les pregunta a los encuestados sobre el dilema de cooperación que les planteamos, las personas que habitan un asentamiento irregular en el sector sur de Chaitén mencionan aproximadamente una persona y media más que los habitantes del norte. Además, el intervalo de confianza ha sido construido de tal manera de que hay una probabilidad del 95\% de que el efecto casual promedio verdadero esté entre 0,06 y 3,14. Es importante mencionar que este intervalo de confianza es ancho, indicando algo de ineficiencia en la estimación. Dicha ineficiencia es confirmada cuando aplicamos ajustes para comparaciones múltiples. En el caso del efecto estimado para cooperación, la corrección de Holm sugiere que dicho efecto es significativo solo para un valor-alpha de 0,10 (test de dos colas). Una forma de lograr más eficiencia es estimar un test de permutaciones con ajuste por covariables que, en simple, considera no solo la asociación entre el tratamiento y el resultado sino también las asociaciones entre este último y las covariables (Rosenbaum et al. 2002). Para la variable cooperación, el test confirma un efecto significativo, con valor $p$ de 0,05 (test de dos colas). ${ }^{15}$

14 Los intervalos de confianza han sido calculados en base a asignación aleatoria y utilizando 10.000 permutaciones.

15 En el caso de este último test, correcciones para comparaciones múltiples no fueron aplicadas, pues no es claro en la literatura el modo como dichas correcciones funcionan en el caso de este tipo de ajustes por covariables. 
Figura 2: Efectos causales de vivir en asentamientos irregulares en confianza y cooperación

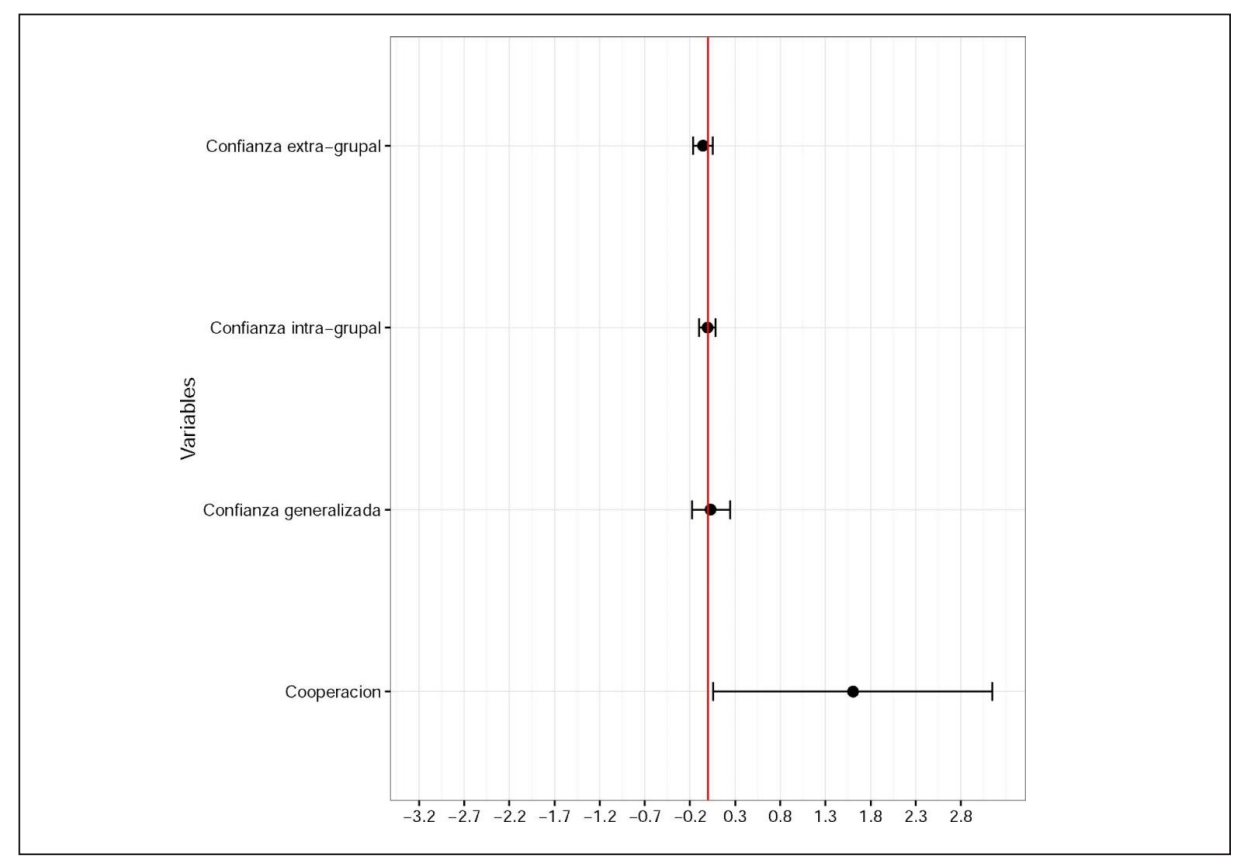

Diferencias norte/sur (ATE)

Fuente: Encuesta de Decisión de Ocupación de Lugares Expuestos a Amenazas Naturales.

Nota: Efectos presentados corresponden a efectos causales promedios estimados (ATEs). Intervalos de confianza estimados en base a inferencia aleatorizada, asumiendo efectos constantes. Debido a casos perdidos, los tamaños muestrales defieren según variables resultados. Estos son 163 para cooperación, 153 para confianza generalizada, 168 para confianza intragrupal, y 157 para confianza extragrupal. La línea vertical de color rojo indica un efecto igual a cero.

Las estimaciones presentadas en la Figura 2 asumen un efecto aditivo y constante. Full Matching nos permite ir más allá y explorar la heterogeneidad del efecto causal, de modo de mejorar el diseño de un estudio observacional. Como primera ilustración de este tema, la Tabla 2 muestra la frecuencia de respuestas para el ítem de cooperación según condición de tratamiento. Como podemos ver, las diferencias entre tratados y controles no son muy grandes, excepto en los valores extremos de la escala de respuesta. En comparación con el sector sur (tratados), los resultados indican que muchos más habitantes del sector norte (control) reportan que no creen que alguna persona cooperaría en el dilema propuesto ( 8 versus 26, es decir, 3,25 veces más a favor del sector norte). La situación se invierte respecto del número máximo de cooperadores (18 versus 10, es decir, 1,8 veces más a favor del sector sur). Este resultado revela una importante heterogeneidad en los efectos asociados con la exposición en cuestión. El análisis del matching confirma dicha heterogeneidad. Al respecto, la Figura 3 muestra un gráfico de cajas que visualiza la distribución de los efectos 
causales de los bloques. ${ }^{16}$ Si bien la mediana es similar al efecto causal promedio estimado, existe un rango de variación importante entre los efectos de cada bloque, teniendo como efectos extremos -10 y 7 . Este resultado sugiere que tanto para controles como para tratados es posible detectar respuesta extremas y, por lo tanto, efectos de gran magnitud. Por ejemplo, vivir en un asentamiento irregular podría generar en algunas personas una disposición extremadamente cooperadora con su comunidad, en el sentido de que vivir en el sector sur está asociado con una mención de 7 cooperadores más que lo mencionado por los habitantes del sector norte.

Tabla 2: Frecuencia de respuestas a pregunta sobre cooperación

\begin{tabular}{lcccccccccc}
\hline & 0 & 1 & 2 & 3 & 4 & 5 & 6 & 7 & 8 & 10 \\
\hline Tratados (n) & 8 & 4 & 8 & 1 & 2 & 17 & 2 & 3 & 5 & 18 \\
\hline Control (n) & 26 & 3 & 4 & 9 & 6 & 23 & 5 & 3 & 6 & 10 \\
\hline
\end{tabular}

Fuente: Encuesta de Decisión de Ocupación de Lugares Expuestos a Amenazas Naturales. Nota: Valores de 0 a 10 indican el número de cooperadores que declara el encuestado.

Figura 3: Heterogeneidad causal del efecto de vivir en asentamiento informal sobre la cooperación

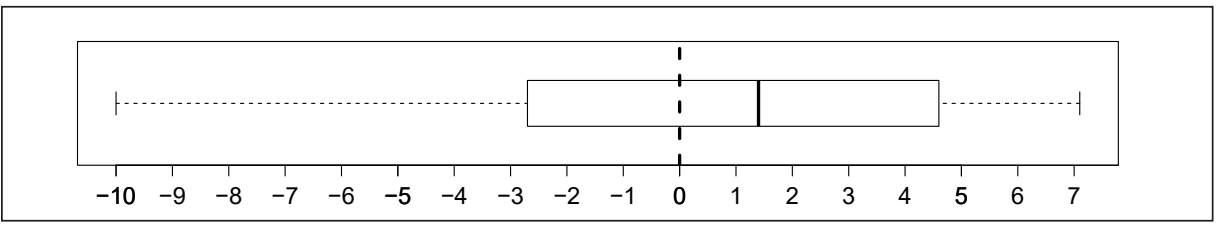

Efecto tratamiento para cada bloque

Fuente: Encuesta de Decisión de Ocupación de Lugares Expuestos a Amenazas Naturales.

Nota: Efectos corresponden a diferencias promedios entre tratados y controles para cada bloque generado por Full Matching.

¿Cuán sensible es el efecto identificado para cooperación respecto de variables no observadas? El análisis de sensibilidad simultáneo presentado en la Tabla 3 puede ayudar a responder esta pregunta. La Tabla 3 presenta tres tipos de valores. Primero, tenemos los valores de $r$, los cuales están en la primera columna de la Tabla 3 y designan las chances u odds que caracterizan a la asociación entre la variable omitida y el tratamiento. Por ejemplo, un $r=1$ corresponde a la situación en donde la chance de recibir el tratamiento es 0,5, es decir, la situación

16 Los efectos para cada bloque fueron estimados como la diferencia (resta) entre la respuesta promedio de tratados y la respuesta promedio de controles para cada bloque. Para una ilustración de este tipo de análisis de heterogeneidad causal ver Rosenbaum (2010) 
ideal de un experimento aleatorizado. En otras palabras, no hay asociación entre la variable omitida y el tratamiento. Un $r=2,5$ significa que la variable omitida aumenta en 2,5 veces las chances de vivir en el sur (tratamiento), lo cual podría ser interpretado como que hay una moderada/fuerte relación entre la variable omitida y el tratamiento. Segundo, la primera fila de la Tabla 3 está compuesta por los valores de A, los cuales caracterizan la asociación entre la variable omitida y el resultado. La interpretación es idéntica a los valores de $r$. Tercero, el resto de los componentes de la Tabla 3 son valores $p$ para cada combinación de $r$ y A bajo la hipótesis nula de no efecto del tratamiento. Tal como se explicó arriba, el objetivo del análisis de sensibilidad es encontrar combinaciones de los parámetros $r$ y A que hacen al efecto bajo análisis no significativo estadísticamente. En el caso de la Tabla 3, todos los valores $p$ son mayores de 0.05 pero hay valores menores de $10 \%$. Es importante señalar que usar este último criterio para evaluar sensibilidad tiene sentido dado el tamaño limitado de nuestra muestra.

Tabla 3: Análisis de sensibilidad para valores seleccionados de A y r en base a residuos de variable resultado

\begin{tabular}{ccccccc}
\hline$r$ & $\mathrm{~A}=1$ & $\mathrm{~A}=1.1$ & $\mathrm{~A}=1.2$ & $\mathrm{~A}=1.5$ & $\mathrm{~A}=2.5$ & $\mathrm{~A}=\infty$ \\
\hline 1 &, 0517 &, 0517 &, 0517 &, 0517 &, 0517 &, 0517 \\
1,1 &, 0517 &, 0527 &, 0539 &, 0563 &, 0618 &, 0785 \\
1,2 &, 0517 &, 0539 &, 0557 &, 0608 &, 0722 &, 1117 \\
1,5 &, 0517 &, 0564 &, 0609 &, 0733 &, 1042 &, 2353 \\
2,5 &, 0517 &, 0625 &, 0736 &, 1073 &, 2054 &, 6557 \\
$\infty$ &, 0517 &, 0903 &, 1403 &, 3357 &, 8561 & 1 \\
\hline
\end{tabular}

Fuente: Encuesta de Decisión de Ocupación de Lugares Expuestos a Amenazas Naturales.

Nota: Análisis de sensibilidad estimado no directamente sobre la variable cooperación, sino sobre los residuos de esta, que fueron obtenidos de un modelo con cooperación como variable dependiente y las covariables observadas como regresores.

Descritos los elementos del análisis de sensibilidad, podemos interpretar los resultados. Las estimaciones de la Tabla 3 indican que la inferencia del efecto de vivir en un asentamiento informal en la cooperación es sensible a una variable omitida que tiene relaciones débiles o moderadas con el tratamiento y con la variable resultado. Por ejemplo, poniéndonos en el escenario más conservador, si asumimos que la variable omitida es casi un perfecto predictor de cooperación $(A=\infty)$, solo basta que este factor no observado aumente las chances de vivir en el sur por $20 \%(r=1,2)$ para que cambiemos nuestras conclusiones sobre el efecto que encontramos para cooperación. Si relajamos el supuesto de casi perfecto predictor, los resultados indican que el efecto pierde significancia estadística si la asociación entre la variable omitida y el tratamiento está caracterizada por una chance de 1,5 y la relación entre el factor no observado y la cooperación 
presenta una chance de 2,5. Resulta importante mencionar que estos valores $p$ no están alejados de los que han sido encontrados comúnmente en ciencias sociales, en donde los valores se mueven en un rango entre 1 y 2 (DiPrete y Gangl 2004; Keele 2010). En suma, el análisis de sensibilidad indica que hay factores no observados que no han sido incluidos en los análisis en base a matching y que podrían ser considerados para lograr resultados más robustos. En la siguiente sección, discutimos las implicancias de este resultado y el resto de nuestra evidencia para la elaboración de un plan de análisis en estudios observacionales.

\section{DISCUSIÓN}

Este artículo tuvo como objetivo presentar una estrategia para generar comparaciones válidas en estudios observacionales. Se discutieron tres elementos que podrían formar parte de dicha estrategia y se los ilustró a través del caso de Chaitén y de los efectos que ha tenido la división de la ciudad en la confianza y la cooperación social de sus habitantes. Los aspectos discutidos en el texto y los resultados del análisis sugieren varios puntos importantes como guías para el desarrollo de planes de análisis para estudios observacionales.

Un primer punto de guía es la centralidad de la comprensión del proceso de autoselección de las unidades en las condiciones del tratamiento. Tal como muestra el artículo, un buen estudio observacional debería poner el foco más en su diseño que en el modelamiento estadístico con el fin de entender el proceso de autoselección y de diagnosticar en qué medida este se acerca o aleja de un diseño aleatorizado. Dado que el foco está en la validación de las comparaciones relevantes, y no en los posibles efectos, se evita la manipulación de resultados, lo que es un aspecto central en la actual discusión sobre planes de análisis en ciencias políticas (Humphreys et al. 2013; Dunning 2016). El desarrollo de un modelo causal puede ser de gran ayuda en dicho diagnóstico, pues nos permite clarificar el tratamiento en cuestión y los supuestos necesarios para identificar sus efectos. En este sentido, es recomendable que la formulación del modelo causal y el planteamiento de un diagnóstico del proceso de selección sean incluidos en planes de análisis de estudios observacionales. Cabe destacar también la gran utilidad del trabajo cualitativo para el diagnóstico de procesos de autoselección en estudios observacionales y de cómo este puede ser incluido en una investigación con diseño mixto que se plantee una pregunta de naturaleza causal. Un área no explorada en este artículo pero que es actualmente de gran interés en ciencias políticas es el uso de métodos cualitativos no solo para diagnosticar el proceso de selección en el tratamiento sino también para indagar en los mecanismos que pueden explicar los efectos encontrados (Acharya et al. 2015).

Un segundo punto de guía refiere al método con el cual se busca validar las comparaciones de un estudio observacional. Este artículo ha ilustrado la utilidad 
de la técnica de Full Matching para lograr tal objetivo. Con ello, hemos presentado al público de habla hispana un método contemporáneo de análisis de efectos causales en contextos no experimentales. Particularmente, nuestro artículo muestra dos grandes ventajas de este método. Primero, independientemente de las excelentes propiedades de balance que logra la metodología, Full Matching nos libra de perder casos, lo cual resulta particularmente importante en estudios que usan tamaños muestrales limitados, pues evita que los test estadísticos pierdan poder. Segundo, el método es particularmente útil para examinar heterogeneidad causal. Al respecto, la investigación sobre consecuencias de desastres naturales indica que el patrón de heterogeneidad identificada en nuestros resultados no sería un resultado aislado. Al parecer, el efecto promedio de los desastres en resultados sociológicos y psicológicos, particularmente de largo plazo, es menor. Sin embargo, hay un grupo de personas para los cuales dichos efectos pueden ser significativos (Zubizarreta et al. 2013). Estadísticos han desarrollado metodologías para analizar este tipo de heterogeneidad causal (Rosenbaum 2007). Estos desarrollos sugieren que un área de investigación futura es desarrollar y ajustar este tipo de metodologías para temáticas de ciencias sociales e incluir el ítem de heterogeneidad causal en planes de análisis.

Finalmente, la discusión planteada en este artículo también indica la centralidad del análisis de sensibilidad en estudios observacionales. Este aspecto es clave para la evaluación de los resultados, en tanto este tipo de diseño asume selección solo sobre variables observadas, lo que es un supuesto fuerte. Análisis de sensibilidad permiten justamente evaluar dicha creencia. En este sentido, los resultados del estudio sugieren la presencia de variables omitidas que podrían estar confundiendo el análisis, ya que detectamos un nivel considerable de sensibilidad para el efecto de un asentamiento informal en la cooperación. Tal como señalamos en el texto, este resultado está en línea con la evidencia sobre el análisis de sensibilidad de estudios observacionales en ciencias sociales. Ahora bien, la sensibilidad detectada en nuestras estimaciones tiene implicancias tanto sustantivas como metodológicas. Respecto de las primeras, un buen diseño observacional debería reflexionar sobre posibles variables omitidas relevantes. ¿Cuáles podrían ser dichos factores? El nivel de conocimiento teórico y del caso de estudio es fundamental para responder esta pregunta. Respecto del caso de Chaitén, tanto la evidencia cualitativa como la información recolectada por nuestra encuesta indican que hay diferencias claras en las preferencias entre los habitantes del sector norte y del sur, que podrían influir en la decisión de ocupar uno u otro lado del río. Análisis de los datos recolectados muestran, por ejemplo, que los residentes del sur aprecian fuertemente el recuperar los bienes perdidos, situación que puede motivarlos a vivir en dicho sector de Chaitén. También el hecho de que los entrevistados vivieron en el norte o en el sur antes de la catástrofe podría estar determinando las preferencias de locación residencial en el Chaitén actual. Lamentablemente, tener información sobre valoraciones no es suficiente para solucionar el problema de variable omitida. Este tipo de valoraciones puede ser fuertemente endógena, lo que, tal como se mencionó, puede introducir sesgo en las estimaciones. 
Este último punto nos lleva a las implicancias metodológicas del análisis de sensibilidad. Siguiendo a Rosenbaum (2010), planes de análisis para estudios observacionales deben intentar examinar las predicciones derivadas de una elaboración teórica y dichas predicciones pueden orientar la investigación. En términos de diseño general de estudios observacionales, una clara elaboración teórica es de gran ayuda para identificar el número de comparaciones a investigar, algunas de las cuales pueden ser usadas, por ejemplo, como placebos. La determinación del número de comparaciones también ayudaría a la especificación de test de hipótesis que corrijan por comparaciones múltiples, evitando de este modo algún tipo de manipulación de valores-p ( $p$-hacking). Para la temática del caso de estudio del presente artículo, esto plantea importantes implicancias teórico-prácticas. El análisis de sensibilidad revela la necesidad de una mayor elaboración teórica de modo de explicar cómo las valoraciones podrían sesgar el efecto de vivir en un asentamiento informal sobre la cooperación. Además, el requerimiento de una mejor comprensión del rol de las valoraciones también plantea la necesidad de desarrollar estudios longitudinales en el campo investigativo de desastres naturales que contemplen en su diseño estrategias de medición de covariables que eviten sesgos postratamiento y que utilicen la información longitudinal, por ejemplo la presencia de eventos como desastres, para construir experimentos naturales (Zubizarreta et al. 2014).

\section{REFERENCIAS}

Abascal, Maria y Baldassarri, Delia. 2015. "Love thy Neighbor? Ethnoracial Diversity and Trust Reexamined". American Journal of Sociology 121(3): 722-782.

Acharya, Avidit, Matthew Blackwell y Maya Sen. 2016. "Explaining Causal Findings Without Bias: Detecting and Assessing Direct Effects". American Political Science Review 110 (3): 512-529.

Berezin, Alan. 2015. “Chaitén: una historia en el lugar". Magallania (Punta Arenas) 43(3): 91106.

Bowers, Jake, Ben B. Hansen y Mark Fredrickson. 2016. "Ritools: Randomization Inference Tools". R package 0.1-13.

Budziak, Jeffrey y Daniel Lempert. 2015. “Assessing Threats to Inference with Simultaneous Sensitivity Analysis: The Case of US Supreme Court Oral Arguments". Political Science Research and Methods: 1-24.

Carn, Simon A., John S. Pallister, Luis Lara, John W. Ewert, Sebastian Watt, Alfred J. Prata, Ronald J. Thomas y Gustavo Villarosa. 2009. "The Unexpected Awakening of Chaitén Volcano, Chile". Eos, Transactions American Geophysical Union 90(24): 205-206.

Cochran, William G. y Paul, S. Chambers. 1965. "The planning of observational studies of human populations". Journal of the Royal Statistical Society. Series A (General) 128(2): 234-266.

Delhey, Jan, Kenneth Newton y Christian Welzel. 2011. "How General is Trust in Most People? Solving the Radius of Trust Problem". American Sociological Review 76(5): 786-807.

DiPrete, Thomas A. y Markus Gangl. 2004. "Assessing Bias in the Estimation of Causal Effects: Rosenbaum Bounds on Matching Estimators and Instrumental Variables Estimation With Imperfect Instruments". Sociological methodology 34(1): 271-310.

Dunning, Thad. 2016. "Transparency, Replication, and Cumulative Learning: What Experiments Alone cannot Achieve". Annual Review of Political Science 19: 541-563. 
Dunning, Thad. 2012. Natural Experiments in the Social Sciences: A design-based Approach. Cambridge: Cambridge University Press.

Espinoza, Adriana E., Claudia E. Espinoza y Anita A. Fuentes. 2015. “Retornando a Chaitén: diagnóstico participativo de una comunidad educativa desplazada por un desastre socionatural". Magallania (Punta Arenas) 43(3): 65-76.

Fernandes, Edésio. 2011. Regularización de asentamientos informales en América Latina, Informe sobre Enfoque en Políticas de Suelo, Lincoln Institute of Land Policy

Gerber, Alan y Donald Green. 2012. Field Experiments: Design, Analysis, and Interpretation. New York: WW Norton.

Hansen, Ben B. 2007. "Flexible, Optimal Matching for Observational studies". R News 7: 18-24.

Hansen, Ben B. 2004. "Full Matching in an Observational Study of Coaching for the Sat". Journal of the American Statistical Association 99(467): 609-618.

Hansen, Ben. B. y Jake Bowers. 2008. "Covariate Balance in Simple, Stratified and Clustered Comparative Studies", Statistical Science 23(2): 219-236.

Hernan, Miguel A. y James M. Robins. 2006. "Estimating causal effects from epidemiological data". Journal of epidemiology and community health 60(7): 578-586.

Hernandez, Bernardo, M. Carmen Hidalgo, M. Esther Salazar-Laplace y Stephany Hess. 2007. "Place attachment and place identity in natives and non-natives". Journal of environmental psychology 27(4): 310-319.

Ho, Daniel. E., Kosuke Imai, Gary King y Elizabeth A. Stuart. 2007. "Matching as Nonparametric Preprocessing for Reducing Model Dependence in Parametric Causal Inference". Political analysis 15(3): 199-236.

Holland, Paul W. 1986. "Statistics and Causal Inference". Journal of the American statistical Association 81(396): 945-960.

Humphreys, Macartan, Raul Sanchez De la Sierra y Peter Van der Windt. 2013. "Fishing, commitment, and communication: A proposal for comprehensive nonbinding research registration". Political Analysis 21(1): 1-20.

Imbens, Guido. W. y Donald B. Rubin. 2015. Causal Inference in Statistics, Social, and Biomedical Sciences. Cambridge: Cambridge University Press.

INE. 2005. Censo 2002, Chile: Ciudades, pueblos, aldeas y caseríos. Tech. rep., Instituto Nacional de Estadísticas, Santiago, Chile. Recuperado de http://www.ine.cl/canales/usuarios/ cedoc_online/censos/pdf/censo_2002_publicado_junio_2005.pdf

James, Gareth, Daniela Witten, Trevor Hastie y Robert Tibshirani. 2013. An Introduction to Statistical Learning, vol. 6. Berlin: Springer.

Keele, Luke. 2010. “An Overview of Rbounds: An R Package for Rosenbaum Bounds Sensitivity Analysis with Matched Data". White Paper. Columbus, OH: 1-15.

Keele, Luke. 2015. "The Statistics of Causal Inference: A View from Political Methodology". Political Analysis 23 (3): 313-335.

Keele, Luke, Corrine McConnaughy y Ismael White. 2012. "Strengthening the Experimenter's Toolbox: Statistical Estimation of Internal Validity". American Journal of Political Science 56(2): 484-499.

Keele, Luke y Rocio Titiunik. 2016. "Natural Experiments Based on Geography". Political Science Research and Methods 4(1): 65-95.

Lara, Luis y Rodrigo Calderón. 2015. "Peligro y riesgo específico asociado al volcán Chaitén: perspectiva geológica de la vulnerabilidad en el entorno de un volcán activo". Magallania (Punta Arenas) 43(3): 27-35.

Luna, Juan Pablo y Hillel David Soifer. 2015. Surveying State Capacity. Latin American Public Opinion Project Insights series N 119.

Major, Jon J. y Luis E. Lara. 2013. “Overview of Chaitén Volcano, Chile, and its 2008-2009 Eruption". Andean Geology 40(2): 196-215.

Ministerio del Interior y Seguridad Publica de Chile. 2014. Diagnóstico estado de la reconstrucción erupción volcán Chaitén 2 de mayo de 2008. Reporte técnico. Santiago: Ministerio del Interior y Seguridad Publica de Chile. Recuperado de http:/ /www.gob.cl/wp-content/uploads/2014/07/Chaitén_V5.pdf 
Observatorio de Ciudades PUC. 2009. Informe final etapa 3 consultoría para el desarrollo de lineamientos estratégicos de reconstrucción / relocalización y plan maestro conceptual post-desastre Chaitén. Reporte técnico. Santiago: Observatorio de Ciudades. Pontificia Universidad Católica de Chile.

ONEMI .2009. Actividad volcánica en Chile. Año 2008. Reporte técnico. Santiago: Ministerio del Interior y Seguridad Publica de Chile. Recuperado de URL= http://repositoriodigitalonemi.cl/web/bitstream/handle/2012/434/Actividad Volcánica en Chile 2008. pdf?sequence $=1$.

Pierson, Thomas C., Jon J. Major, Alvaro Amigo y Hugo Moreno. 2013. “Acute Sedimentation Response to Rainfall Following the Explosive Phase of the 2008-2009 Eruption of Chaitén Volcano, Chile". Bulletin of Volcanology 75(5): 1-17.

Rose, Sherri. 2015. "Targeted learning for pre-analysis plans in public health and health policy research". Observational Studies 1: 294-306.

Rosenbaum, Paul R. 1984. "The Consequences of Adjustment for a Concomitant Variable that has been Affected by the Treatment". Journal of the Royal Statistical Society. Series A (General) 147 (5): 656-666.

Rosenbaum, Paul R. 1991. "A Characterization of Optimal Designs for Observational Studies". Journal of the Royal Statistical Society. Series B (Methodological) 53 (3): 597-610.

Rosenbaum, Paul R. 2007. "Confidence Intervals for Uncommon But Dramatic Responses to Treatment". Biometrics 63(4): 1164-1171.

Rosenbaum, Paul R. 2010. Design of Observational Studies. Berlin: Springer.

Rosenbaum, Paul R. 2015. "Two R Packages for Sensitivity Analysis in Observational Studies". Observational Studies 1: 1-17.

Rosenbaum, Paul R. 2002. "Covariance Adjustment in Randomized Experiments and Observational Studies". Statistical Science 17(3): 286-327.

Rosenbaum, Paul R. y Donald R. Rubin. 1983. "The Central Role of the Propensity Score in Observational Studies for Causal Effects". Biometrika 70(1): 41-55.

Samii, Cyrus. 2016. "Causal empiricism in quantitative research". The Journal of Politics 78(3): 941-955.

Stuart, Elizaneth A. y Kerry M. Green. 2008. “Using Full Matching to Estimate Causal Effects in Nonexperimental Studies: Examining the Relationship Between Adolescent Marijuana Use and Adult Outcomes". Developmental psychology 44(2): 395-406.

Ugarte, Ana María, y Marcela Salgado. 2014. "Sujetos en emergencia: acciones colectivas de resistencia y enfrentamiento del riesgo ante desastres; el caso de Chaitén, Chile", Revista Invi 29(80): 143-168.

Zubizarreta, José R., Magdalena Cerda y Paul R. Rosenbaum. 2013. “Effect of the 2010 Chilean Earthquake on Post-traumatic Stress Reducing Sensitivity to Unmeasured Bias Through Study Design". Epidemiology 24(1): 79-87.

Zubizarreta, José R., Dyan S. Small y Paul R. Rosenbaum. 2014. "Isolation in the Construction of Natural Experiments". The Annals of Applied Statistics 8(4): 2096-2121.

Luis Maldonado Navarro: Instituto de Sociología, Pontificia Universidad Católica de Chile; Centro Nacional de Investigación para la Gestión Integrada de Desastres Naturales (CIGIDEN). Correo electrónico: lmaldona@uc.cl

Edmundo Kronmüller Rioseco: Escuela de Psicología, Pontificia Universidad Católica de Chile; Centro Nacional de Investigación para la Gestión Integrada de Desastres Naturales (CIGIDEN); Centro de Estudios Interculturales e Indígenas (CIIR). Correo electrónico: ekr@uc.cl

Ignacio Gutiérrez Crocco: Centro Nacional de Investigación para la Gestión Integrada de Desastres Naturales (CIGIDEN). Correo electrónico: iagutierrezcrocco@gmail.com 


\section{APÉNDICE}

En ciencias sociales, el modelo causal de Neyman-Rubin representa un marco conceptual ideal para estudiar efectos. ${ }^{17}$ En este modelo, cada individuo $i$ tiene dos resultados potenciales pero solo un resultado factual o realizado. Los resultados potenciales representan el comportamiento del individuo en presencia y ausencia de un tratamiento, y el resultado observado depende del estatus de tratamiento observado. Sea D el tratamiento, en donde 1 indica el grupo de tratamiento que refiere a vivir en un asentamiento irregular y 0 denota el grupo de control, el cual representa la condición de vivir en un asentamiento formal. Y es la variable resultado o dependiente, la que medirá indicadores de cooperación y confianza social. El resultado observado es una función de los resultados potenciales y la asignación del tratamiento de tal modo que $Y_{i}=D_{i} Y_{1}$ $(1)+\left(1-D_{i}\right) Y_{i}(0)$. Esta notación muestra, por ejemplo, los resultados potenciales para los tratados, los cuales podrían ser: a) el nivel (factual) de cooperación o confianza social si se es tratado, es decir, si el individuo vive en un asentamiento irregular; b) el nivel (contrafactual) de cooperación o confianza social si un tratado viviera en un asentamiento formal.

Los escenarios factuales y contrafactuales dejan en evidencia lo que es conocido como el problema fundamental de la inferencia causal (Holland 1986). Dicho problema refiere a la imposibilidad de observar los resultados factuales y contrafactuales de un individuo al mismo tiempo. Una solución a este problema es focalizarse en los estimadores poblacionales. Al respecto, una opción estándar es usar promedios. Específicamente, tenemos $E\left[Y_{i}(1)\right]$, que refiere al valor esperado (promedio) de la variable resultado $\mathrm{Y}$ para todos los sujetos en la condición de tratamiento, y $E\left[Y_{i}(0)\right]$ que denota el valor esperado para todos los sujetos en la condición de control. Con estos elementos se define el efecto causal del siguiente modo

$$
E[Y i(1)]-E[Y i(0)]
$$

(1) compara el valor promedio de cooperación o confianza para todos los sujetos en la situación de que ellos fueran tratados y en la condición de control. Ciertamente, (1) no es suficiente para solucionar el problema fundamental de la inferencia causal, pues nosotros más bien observamos $E\left[Y_{i}(1) \mid D_{i}=1\right]$ y $E\left[Y_{i}(0) \mid D_{i}=0\right]$. En consecuencia, para identificar el efecto a nivel poblacional necesitamos hacer supuestos que hagan equivalentes las condiciones que observamos con las condiciones del efecto causal en (1). Así tenemos que

$$
\begin{gathered}
E[Y i(1) \mid \mathrm{Di}=1]=E[\mathrm{Y}(1) \mid \mathrm{Di}=0]=E[\mathrm{Yi}(1)] \\
E[\mathrm{Y}(0) \mid \mathrm{Di}=0]=E[\mathrm{Y}(0) \mid \mathrm{Di}=1]=E[\mathrm{Y}(0)]
\end{gathered}
$$


Las expresiones (2) y (3) son consideradas en la literatura de inferencia causal como parte del supuesto de independencia o intercambiabilidad (Imbens y Rubin, 2015), que formalmente puede ser expresado como ( $\left.Y_{i}(1), Y_{i}(0)\right)$ independiente de $\mathrm{D}_{\mathrm{i}}$. Básicamente independencia significa que si (2) y (3) se cumplen, podemos usar el grupo de control como contrafactual del grupo de tratamiento y viceversa. Asignación aleatoria es justamente el proceso físico que logra tal intercambiabilidad de los grupos. En el caso de nuestro estudio observacional, tenemos que, si independencia fuera un supuesto válido, los habitantes del sector norte y del sector sur serían comparables entre sí en todas las características observadas y no observadas relevantes para la asignación del tratamiento. Con estos elementos podemos definir el efecto causal que se investiga empíricamente del siguiente modo

$$
A T E=E[Y(1) \mid \mathrm{Di}=1]-E[\mathrm{Y}(0) \mid \mathrm{Di}=0]
$$

en donde ATE significa efecto causal promedio (average causal effect). Como se puede notar, (4) es el clásico estimador de diferencias de medias, el cual identifica el efecto causal definido en (1) si el supuesto central de independencia se cumple. ${ }^{18}$ La situación típica en donde este es el caso es la de un experimento aleatorizado, pues la asignación aleatoria, como ya se dijo, asegura que los grupos de tratados y controles sean intercambiables. En el caso de estudios observacionales, existe una versión de este supuesto que se llama independencia condicional. Si asumimos que Ui es un vector de variables no observadas, este supuesto puede ser expresado como $D_{i}$ independiente de $\left(Y_{i}(1), Y_{i}(0), U_{i}\right) \mid M_{i}$. Esto significa que podemos demandar que el tratamiento es independiente de los resultados y de las variables no observadas, es decir demandamos asignación aleatoria del tratamiento, si condicionamos por M. Este supuesto también se conoce como asignación del tratamiento fuertemente ignorable dado $M$. En base al supuesto de independencia condicional, el efecto causal promedio es el siguiente

$$
A T E=E\left[Y i(1) \mid D_{i}=1, \mathrm{M}\right]-E[Y(0) \mid \mathrm{Di}=0, \mathrm{M}]
$$

$\mathrm{M}$ refiere a todas las covariables que influyen tanto en el tratamiento como en la variable resultado. En el caso de matching, M corresponde a estratos que son generados por el procedimiento de emparejamiento de tratados y controles.

18 Un segundo supuesto es necesario para identificar ATE. Este se llama stable-unit-treatmemt assumption (SUTVA) y estipula que el mecanismo usado para asignar al tratamiento no es relevante para la consistencia del tratamiento y que no hay interferencia entre las unidades experimentales. Siguiendo la práctica habitual de los estudios en ciencias sociales, nuestra estrategia de identificación causal asume SUTVA. 
